\title{
Band structure effects in Auger neutralization of He ions at metal surfaces
}

\author{
D. Goebl, ${ }^{1, *}$ Diego Valdés, ${ }^{2}$ E. Abad, ${ }^{2}$ R. C. Monreal, ${ }^{2}$ D. Primetzhofer,${ }^{1}$ and P. Bauer ${ }^{1}$ \\ ${ }^{1}$ Institut für Experimentalphysik, Johannes Kepler Universität Linz, A-4040 Linz, Austria \\ ${ }^{2}$ Departamento de Física Teórica de la Materia Condensada C5, Universidad Autónoma de Madrid, E-28049 Madrid, Spain
}

(Received 22 June 2011; revised manuscript received 30 August 2011; published 18 October 2011)

\begin{abstract}
A comparative study of Auger neutralization (AN) of He ions at noble metal surfaces is presented in order to reveal how the electronic structure of the sample influences this charge exchange process. Comparison of calculated ion fractions to experimental data obtained in low energy ion scattering (LEIS) shows that good agreement is achieved only if the relevant aspects of the He-metal interaction are properly taken into consideration. For instance AN depends sensitively on the distance-dependent position of the projectile level, which varies significantly when considering different target materials.
\end{abstract}

DOI: 10.1103/PhysRevB.84.165428

PACS number(s): 79.20.Rf, 34.70.+e, 68.47.De, 68.49.Sf

\section{INTRODUCTION}

When a positive ion approaches a surface one can distinguish between two different types of processes that lead to charge exchange: two-electron processes such as Auger neutralization (AN) or Auger de-excitation and one-electron transitions, i.e., resonant neutralization $(\mathrm{RN})$ and resonant ionization (RI). These processes have been under investigation for many decades, ${ }^{1-3}$ and much progress has been made recently. ${ }^{4-8}$ Due to their level of complexity, a complete understanding of these processes has not yet been reached. To proceed one step further toward this aim, the present study investigates how the electronic properties of the target influence $\mathrm{AN}$ of $\mathrm{He}^{+}$ions in low energy ion scattering (LEIS) where single scattering events by a large angle prevail. This is not only of fundamental interest but also of vital importance in manifold applications, especially for surface analysis techniques such as Auger electron spectroscopy, ${ }^{9-12}$ ion scattering under grazing incidence, ${ }^{13}$ and LEIS. ${ }^{14,15}$

LEIS is a common surface analysis technique, which is sensitive to the outermost atomic layers of the sample under investigation when noble gas ions are used as projectiles. ${ }^{14,15}$ Typically primary beam energies $E$ are in the range of 0.5 to $10 \mathrm{keV}$, and the scattering angle $\vartheta$ is chosen between $100^{\circ}$ and $170^{\circ}$. The field of application is mostly analysis of surface structure or surface composition. In order to obtain quantitative results with LEIS, detailed knowledge of the charge exchange processes at surfaces is of crucial importance. ${ }^{16,17}$

In LEIS charge exchange is mostly governed by two different processes. First, AN is possible whenever an occupied metal state is energetically situated above the empty ion state. The process is a two-electron process in which an electron from a metal state is scattered to the unoccupied ion state, while the gain in potential energy is transferred to the electronic system of the metal, which can either lead to the excitation of a single electron or a plasmon. Second, resonant charge transfer is possible whenever an empty projectile level is in resonance with an occupied target level (RN) or vice versa (RI). $\mathrm{RN}$ of $\mathrm{He}^{+}$is not possible at sufficiently large ion-surface distances when the He 1s level is well below the bottom of the conduction band and excited He states are above the Fermi level, as is the case for the vast majority of elemental surfaces. With decreasing projectile-surface distance the $\mathrm{He}$ 1s level undergoes a significant energy shift when the $\mathrm{He}$ level is promoted due to its interaction with bound states of target atoms. ${ }^{13,18}$ In consequence resonant transitions become possible whenever the distance between the ion and an atom is smaller than a critical distance, $r_{0}$. For a given value of $\vartheta$, the minimum distance in the collision becomes smaller than $r_{0}$ for energies exceeding a threshold energy $E_{0}$. For $E<E_{0}$ neutralization is basically due to $\mathrm{AN}$; at the corresponding energies, quantum resonant processes due to the finite width of the He level and nonadiabatic effects are negligible.

AN is very well understood on a qualitative basis by now, but the specific influence of the electronic structure of a target on this process is still a matter of current investigations. Experimental results on neutralization of $\mathrm{He}^{+}$at $\mathrm{Ag}$ surfaces at grazing angles of incidence exhibited an unexpected dependence of the ion fraction on the crystallographic orientation of the surface. This finding was explained by the fact that for different Miller indices the jellium edge is located at different distances to the surface planes, which contain the outermost atomic layers. ${ }^{19,20}$ Thorough investigations revealed that a theory of AN beyond the jellium model is required to explain existing experiments on noble metals and to be able to make quantitative predictions there. To this end, a theory of AN was formulated using a Linear Combination of Atomic Orbitals (LCAO) basis for the metal electrons. ${ }^{21}$ This model successfully explains experimental results for the neutralization of $\mathrm{He}$ ions at $\mathrm{Al}$ and $\mathrm{Ag}$ surfaces in the grazing scattering regime. ${ }^{22-24}$

Recent experiments for $\mathrm{He}^{+}$ions scattered from $\mathrm{Cu}$ and $\mathrm{Au}$ crystals in the AN regime of LEIS exhibited a pronounced dependence of the neutralization probability on the surface orientation, similarly as discussed above for grazing collisions. ${ }^{25,26}$ Analog investigations for Ag crystals did not result in a pronounced orientation dependence; ${ }^{26,27}$ therefore, in a recent effort the LCAO model was adopted to also describe $\mathrm{AN}$ in the large angle scattering geometry. First results obtained for $\mathrm{He}^{+}$scattered from $\mathrm{Ag}$ (110) in different azimuth directions were in good agreement with experimental data. ${ }^{28}$ The aim of the present work is to analyze the experimental ion fraction of $\mathrm{He}^{+}$scattered off different surfaces of the three noble metals $\mathrm{Cu}, \mathrm{Ag}$, and $\mathrm{Au}$ in the $\mathrm{AN}$ regime in LEIS by use of this model. A unified picture emerges from this analysis: at close distances to the surface, where the neutralization occurs, 
the process is controlled by the $d$-electrons of the metal; the distance-dependent position of the He 1s level plays a dominant role for obtaining good agreement with the measured values of the ion fractions. Atomic units $\left(e=\hbar=m_{e}=1\right)$ will be used unless otherwise stated.

\section{THEORY}

\section{A. AN rate}

$\mathrm{AN}$ is commonly described by the corresponding transition rate $\Gamma$. A theory to calculate $\Gamma$ for ions in front of metal surfaces was developed, taking into account the crystal structure of the surface. ${ }^{21-23}$ Here, only the main features of this theory will be summarized. The basic idea is to separate the metal electron which neutralizes the ion from the metallic excitations taking the excess of potential energy. The wave function of the neutralizing electron is described using a LCAO basis, while the metal excitations are described within the jellium model. Then, the AN rate can be expressed as

$$
\begin{aligned}
\Gamma\left(\vec{r}_{a}\right)= & 2 \sum_{R, R^{\prime}, \alpha, \alpha^{\prime}} \int_{\omega_{b}}^{\omega_{b}+E_{F}} d \omega \int \frac{d \vec{q}_{\|}}{(2 \pi)^{2}} \int d z \int d z^{\prime} \\
& \times\left(-\operatorname{Im} \chi\left(\vec{q}_{\|}, \omega, z, z^{\prime}\right)\right) V_{\alpha, R}\left(z, \vec{q}_{\|}\right) \\
& \times V_{\alpha^{\prime}, R^{\prime}}^{*}\left(z^{\prime}, \vec{q}_{\|}\right) \rho_{R, \alpha, R^{\prime}, \alpha^{\prime}}\left(\omega+E_{a}\left(\vec{r}_{a}\right)\right) .
\end{aligned}
$$

In this equation $\chi\left(\vec{q}_{\|}, \omega, z, z^{\prime}\right)$ is the response function of the metal surface to an excitation of energy $\omega$ and parallel momentum $\vec{q}_{\|}$, which will be calculated for a jellium surface. $\omega_{b}$ is the minimum excitation energy, corresponding to an electron at the bottom of the conduction band neutralizing the ion. In AN the excitation originates from Coulomb interaction between the ion and the metal electron, which neutralizes the ion. This process is described by matrix elements $V_{\alpha, R}$ which, after Fourier transformation of the coordinates parallel to the surface, read

$$
V_{\alpha, R}\left(z, \vec{q}_{\|}\right)=\frac{2 \pi}{q_{\|}}\left\langle\phi_{a}\left(\vec{r}_{1}-\vec{r}_{a}\right)\left|e^{i \vec{q}_{\|} \vec{\rho}_{1}} e^{-q_{\|}\left|z-z_{1}\right|}\right| \phi_{\alpha}\left(\vec{r}_{1}-\vec{R}\right)\right\rangle .
$$

In Eq. (2) $\phi_{a}\left(\vec{r}_{1}-\vec{r}_{a}\right)$ and $\phi_{\alpha}\left(\vec{r}_{1}-\vec{R}\right)$ are localized orbitals representing the $1 \mathrm{~s}$ orbital of the He atom placed at a point $\vec{r}_{a}$ with respect to the substrate unit cell and the atomic orbital $\phi_{\alpha}$ of a substrate atom at the lattice point $\vec{R}$, respectively. These are orthonormal orbitals constructed from the purely atomic orbitals of independent He and target atoms by means of Löwdin's prescription. ${ }^{29} \rho_{R, \alpha, R^{\prime}, \alpha^{\prime}}$ is the substrate density of states (DOS) projected to the localized basis. In this work the response function is calculated within the jellium model, with the jellium edge placed at half an interlayer distance in front of the first atomic layer. The possibility that a $s$ or $d$-electron is excited in the Auger process is taken into account by means of an effective mean electron radius $r_{s}(\omega)$, which is calculated from the mean number of excited electrons per atom $n(\omega)$ measured in optical experiments ${ }^{30-32}$ via

$$
\frac{4 \pi r_{s}^{3}(\omega)}{3}=\frac{V}{n(\omega)}
$$

Here $V$ corresponds to the volume of the primitive unit cell of the metal. For the unperturbed and unreconstructed surfaces DOS have been calculated using the equation

$$
\begin{aligned}
\rho_{R, a, R^{\prime}, a^{\prime}}(\omega) & =-\frac{1}{\pi} \operatorname{Im}\left(G_{R, a, R^{\prime}, a^{\prime}}(\omega)\right) \\
& =-\frac{1}{\pi} \operatorname{Im}\left[\left((\omega-i \eta) I-H_{R, a, R^{\prime}, a^{\prime}}\right)^{-1}\right],
\end{aligned}
$$

where the Hamiltonian $H$ is calculated by means of the $a b$ initio density functional theory (DFT) local orbital code FIREBALL, ${ }^{33,34}$ based on the ideas by Sankey and Niklewski. ${ }^{35}$ In order to avoid numerical instabilities and to smooth the peak-like DOS obtained in FIREBALL, a small quantity $\eta$ is introduced $(\eta=0.1)$. The employed DOS are in good agreement with other $a b$ initio calculations.

Note that in Eq. (1) the energy level $E_{a}\left(\vec{r}_{a}\right)$ of the ion (with respect to the Fermi level) is an upper limit for the excitation energy. This is of importance for the results, as will be shown in the Results and Discussion section. Other parameters that enter Eq. (1) are the work function, DOS, and effective number of electrons, but they are of minor importance.

To investigate the relationship between electronic structure and $\Gamma$, a closer look is taken at the AN rates at the on-top position of different noble metal surfaces. The on-top position corresponds to $\mathrm{a} \mathrm{He}^{+}$ion approaching the surface on top of a surface atom. In the case of normal incidence and assuming exclusively single scattering, the rates for the on-top position are of particular interest, since neutralization of the ion, prior to the scattering event, is almost entirely described by the on-top rate. ${ }^{28}$ Figure 1 presents the on-top rates for $\mathrm{He}^{+}$approaching $\mathrm{Cu}(111), \operatorname{Ag}(111)$, and $\mathrm{Au}(111)$ surfaces, respectively. The total rate (black squares) is decomposed into contributions from different orbitals: $s$-orbitals (blue triangles) and $d$-orbitals (green diamonds). These correspond to the terms $\alpha=\alpha^{\prime}=$ $s$-orbital and $\alpha=\alpha^{\prime}=d$-orbital of Eq. (1), respectively. Terms crossing orbitals of different symmetry are negligible. Additionally the total contribution of the on-top atom is shown (red circles, dashed). The general behavior of all three surfaces is very similar. As one can see, the total AN rate is governed by transitions from $d$-orbitals for $z$ distances below 2 a.u.; at $z=1$ a.u., contributions from $d$-orbitals are higher than contributions from $s$-orbitals by at least a factor of 3 . The $d$-orbital contributions arise almost exclusively from the on-top atom; $d$-orbital contributions from neighbor atoms are negligible. Evidence for this is given by the position of the peak of $d$-orbital contributions, which correlates with the position of maximum overlap of the He $1 \mathrm{~s}$ level with the $d_{3 z^{2}-r^{2}}$ orbital of the on-top atom.

Contributions from $s$-orbitals originate from the on-top atom and its nearest neighbors in the first and second layer. The relative contributions from neighbors increase with increasing ion-surface distance. Additionally one can see the influence of different lattice parameters on $\mathrm{AN}$ : $\mathrm{Cu}$ as opposed to $\mathrm{Au}$ and $\mathrm{Ag}$ has a notably smaller lattice parameter $(\mathrm{Cu}: 3.6147 \AA$, Ag: $4.0857 \AA$, Au: $4.0783 \AA$ ) and consequently a smaller distance between the on-top atom and its neighbors. As a result, the contribution from neighbor atoms, which is mostly due to $s$-orbital contributions, becomes important at smaller ion-surface distances. 


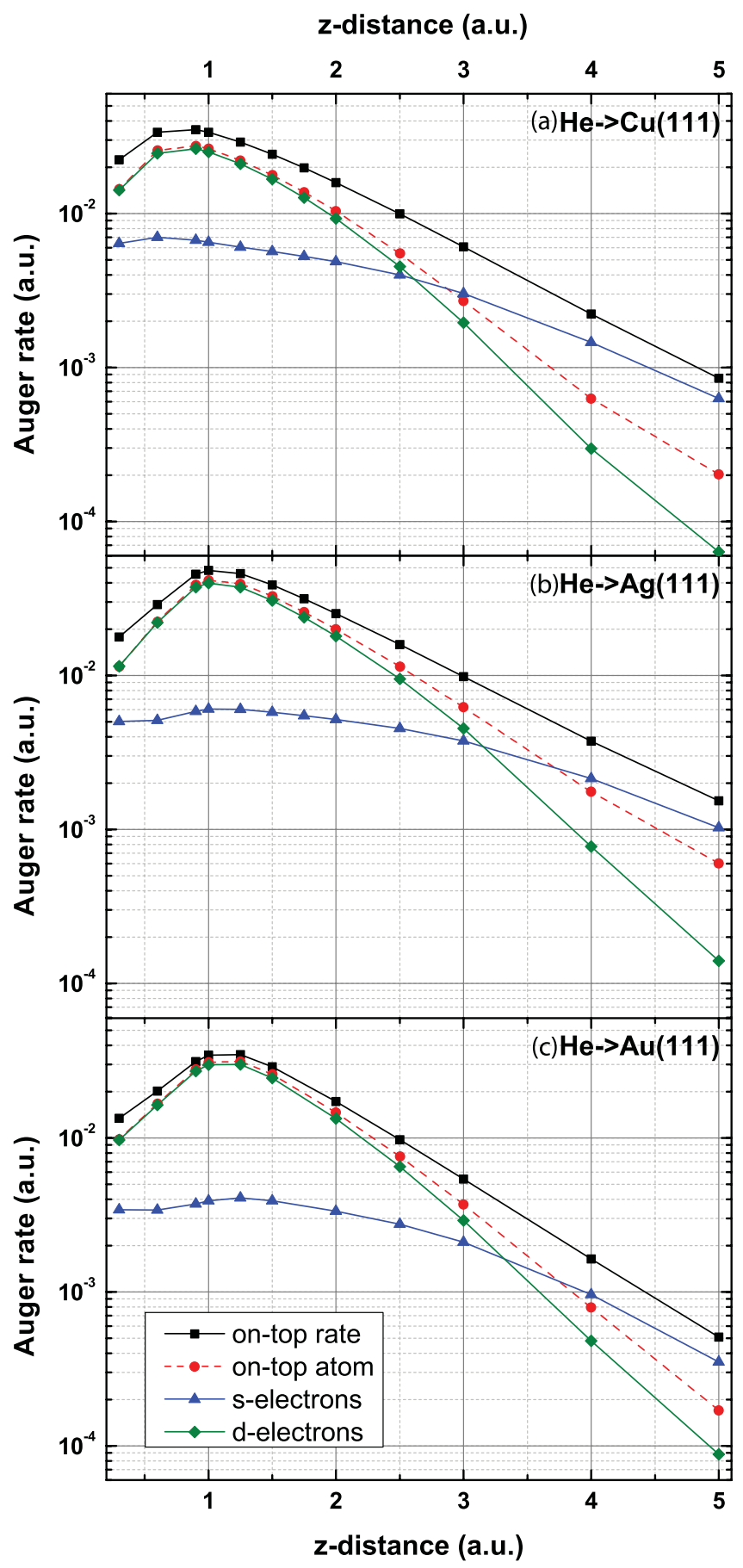

FIG. 1. (Color online) AN rates for $\mathrm{He}^{+}$approaching (a) $\mathrm{Cu}(111)$, (b) $\mathrm{Ag}(111)$, and (c) $\mathrm{Au}(111)$ on top of a surface atom. The rate is decomposed into contributions from the $s$-electrons (blue upward triangles) and from the $d$-electrons (green diamonds). Additionally, contributions from the on-top atom are shown (red circles).

Given the fact that in the LEIS regime the turning point of typical trajectories is well below 2 a.u. in front of the first atomic layer and that for large ion-surface distances the AN rate decays exponentially with increasing distance, ${ }^{23}$ one can infer that the neutralization process is determined by AN rates for $z<2$ a.u. In this region AN of $\mathrm{He}$ at noble metal surfaces is governed by $d$-orbital electrons of the scattering partner, which makes AN at these surfaces a very localized process. This behavior is in strong contrast to the behavior found for Al surfaces. ${ }^{21}$ Here, the contributions originating from the nearest neighbors are larger than the contributions from the on-top atom at all distances investigated.

\section{B. He 1s level shift}

Several effects lead to the modification of the He 1s level in front of a surface: at large distances, the level shift can be described by the classical image potential. ${ }^{13}$ At smaller distances chemical effects may influence the position of the level drastically: ${ }^{18}$ the presence of metal states with energies above the He 1s state leads to an increase in the binding energy when the $\mathrm{He}^{+}$ion reaches a certain distance from the surface. At even smaller distances, the interactions with core electrons can lead to a promotion of the level above the Fermi level. Thus, the level shift as a function of ion surface distance depends on the electronic configuration of projectile and target. Theoretical investigations of this effect were mostly concerned with $\mathrm{H}^{+}$or $\mathrm{He}^{+}$projectiles scattered from $\mathrm{Al}$ surfaces also with the background of explaining charge exchange in resonant transitions. ${ }^{18,36-43}$ Since the level shift is not directly observable, experimental data on this effect are very scarce: Wethekam et al. were able to deduce the level shift of $\mathrm{He}^{+}$in front of $\mathrm{Al}$ based on the deviation from the specular reflection angle in grazing scattering. ${ }^{44}$ Lancaster et al. inferred the level shift from the high-energy cut-off in Auger electron spectra. ${ }^{9}$

The effect of the level shift on AN was already discussed in the pioneering work by Hagstrum. ${ }^{3}$ In these investigations an upward shift of the He 1s level by $2 \mathrm{eV}$ was identified as a remarkably universal figure for various $\mathrm{He}^{+}$-metal combinations when considering low perpendicular energies (several $\mathrm{eV}$ ) of the projectile. This value is also confirmed by recent investigations of $\mathrm{He}^{+}$scattered from $\mathrm{Ag}(111)$ and $\mathrm{Ag}(110)$, where agreement between experiment and theory was obtained assuming a level shift of $2 \mathrm{eV}^{45}$ Experiments for $\mathrm{He}^{+}$scattered from various $\mathrm{Al}$ surfaces yielded, depending on the perpendicular energy, the shift of the He 1s level is in the range between $-1 \mathrm{eV}$ and $2 \mathrm{eV}^{24}$ All these experiments were conducted in grazing scattering geometry, where the perpendicular energy of the projectiles is small. In contrast due to the large perpendicular energy in the LEIS regime, typical trajectories comprise a backscattering collision with a single atom characterized by a very small distance of closest approach. In this close collision the electronic interaction between projectile and collision partner is very strong, leading to a pronounced variation of the $\mathrm{He} 1 \mathrm{~s}$ level as a function of distance.

Unfortunately detailed calculations concerning the $\mathrm{He} 1 \mathrm{~s}$ level shift for $\mathrm{He}^{+}$in front of noble metal surfaces are not available. Hence, two different approaches, both with a free parameter, are employed in this investigation in order to mimic the He 1s level shift.

(i) The "hard wall" level shift is characterized by a constant value of the energy level and a step-like rise at a position corresponding to $r_{0}$; here, the free parameter is the energy position of the constant level. The hard wall simulates the pronounced promotion of the level at very short distances. 


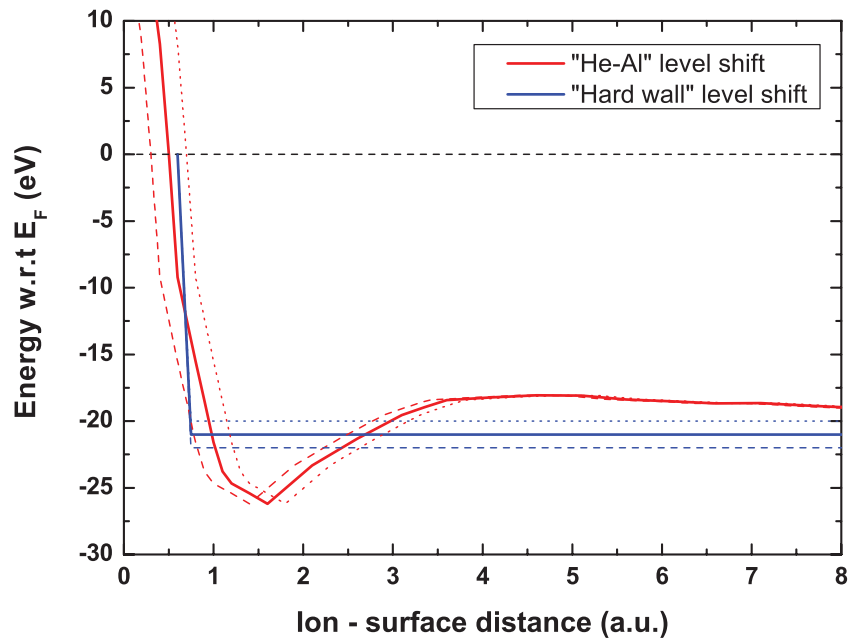

FIG. 2. (Color online) Position of the He 1s level as a function of ion-surface distance according to two different models: "He-Al" level shift (red) and "hard wall" level shift (blue). The hard-wall level shift is depicted for three different level positions, i.e., $-20 \mathrm{eV}$ (dotted), $-21 \mathrm{eV}$ (solid), and $-22 \mathrm{eV}$ (dashed). The depicted He-Al level shifts correspond to displacements of the original He-Al shift ${ }^{18}$ toward the surface by 0.2 a.u. (dotted), 0.4 a.u. (solid), and 0.6 a.u. (dashed), respectively.

(ii) The "He-Al" level shift is based on the level shift recently calculated for $\mathrm{He}$ in front of an $\mathrm{Al}$ surface. ${ }^{18}$ In order to adapt the He-Al level shift to the present systems, the level shift is displaced by a certain amount in distance toward the surface. This is motivated by the fact that, as explained previously, the deviation from the image shift is caused by the interaction of $\mathrm{He}$ with the conduction band electrons. In the noble metals the density of sp-electrons is smaller than in $\mathrm{Al}$, and the effect of the $d$-band electrons should operate at shorter distances. These two effects cooperate in the way of moving the interaction region towards the surface.

Figure 2 presents both approaches: the hard-wall level shift (blue) with the $\mathrm{He}$ level at $-21 \mathrm{eV}$ (solid), $-20 \mathrm{eV}$ (dotted), and $-22 \mathrm{eV}$ (dashed) w.r.t. $E_{\mathrm{F}}$, and the He-Al level shift (red) displaced by 0.4 a.u. (solid), 0.2 a.u. (dotted), and 0.6 a.u. (dashed) from the original He-Al shift towards the surface. It will be shown in the next section that both approaches produce almost identical results.

\section{Analysis of ion fractions}

Results obtained with this model are compared to experimentally obtained ion fractions. The ion fraction $P^{+}$is defined as the fraction of projectiles which reach the detector in a positively charged state $P^{+}=A^{+} /\left(A^{+}+A^{0}\right)$. When charge exchange is exclusively due to $\mathrm{AN}$, the relationship between $P^{+}$and the $\mathrm{AN}-$ rate $\Gamma$ is based on the following rate equation: $d P^{+}=-P^{+} \Gamma d t$. From this expression it is possible to derive $P^{+}$for the incoming/outgoing part of the trajectory

$$
P_{i}^{+}=\exp \left(-\int_{t_{i, 1}}^{t_{i, 2}} \Gamma\left(\vec{r}_{a}(t)\right) d t\right),
$$

where $i$ stands for in and out, respectively; the instants $t_{i, 1}$ and $t_{i, 2}$ refer to starting point of the trajectory and the turning point, respectively. AN rates were calculated for specific points in front of the surface, $\Gamma(x, y, z)$, and linear interpolation was used to determine the rate for all ion positions along its trajectory. Trajectories, required to deduce $\mathrm{P}^{+}$using Eq. (3), were calculated by means of the molecular dynamics (MD) simulation program KALYPSO ${ }^{46}$ under the premise of exclusive single scattering. The interaction between projectile and target was described by the universal potential by Ziegler, Biersack and Littmark (ZBL). ${ }^{47}$ The use of other interatomic potentials, e.g., Thomas-Fermi-Moliere with reasonable values for the screening length, does not change the resulting ion fraction in a significant way. The reason is that-in the energy range investigated in this work-the turning points of the different trajectories are in the region of distances where the AN rates start to decrease. Thus, only a minor impact of the position of the turning point on the AN probability is found. It is observed that the effects of the scattering potential are definitively much smaller than the effects of the He-level shift, which will be discussed in the next section.

The polycrystalline samples, which were investigated in this work, were approximated as a surface with randomly oriented (111) domains. ${ }^{48}$ Consequently the ion fraction was obtained as an average over trajectories scattered from a (111) surface with normal incidence and exiting in arbitrary azimuth directions.

The equivalent information from experimental data is deduced from scattered yields $A^{+}$and $A^{0}$ (see previous discussion). When $P^{+}$is plotted as a function of $1 / v_{\perp}=$ $1 / v_{\perp, \text { in }}+1 / v_{\perp, \text { out }}$, a single-exponential fit to the $P^{+}=$ $\exp \left(-v_{c} / v_{\perp}\right)$ data yields the characteristic velocity $v_{c}$, which is related to $\Gamma$ via $v_{c}=\int \Gamma d z$. Thus, $v_{c}$ is a convenient measure of neutralization efficiency for a given surface-projectile combination.

Experiments and simulations for (100) and (110) surfaces were performed in double alignment geometry, which corresponds to normal incidence and exit in [001] and [1112] azimuth direction, respectively.

The experimental data to which the theoretical calculation is compared was obtained with the time-of-flight LEIS set-up ACOLISSA. ${ }^{49}$ Therefore, calculations were performed for a scattering angle of $129^{\circ}$. In the calculation an acceptance angle of $\pm 1.6^{\circ}$ was used (experiment: $\pm 0.46^{\circ}$ ). Unless otherwise noted, the incident beam was aligned with the surface normal, and primary energies were chosen below or slightly above the threshold energy for resonant charge exchange in close collisions, $E_{0}$. Energies slightly above $E_{0}$ are calculated to facilitate the determination of the single-exponential fit through the calculated points. A common feature of the noble metals is a comparatively high value of $E_{0}(\mathrm{Cu}: 2 \mathrm{keV}, \mathrm{Ag}: 1.25$ $\left.\mathrm{keV}, \mathrm{Au}: 1 \mathrm{keV}^{25,26}\right)$. This allows one to investigate $\mathrm{AN}$ of $\mathrm{He}^{+}$ ions at noble metal surfaces in a rather wide range of energies.

\section{RESULTS AND DISCUSSION}

With the AN rates depicted in Fig. 1 and the simulated trajectories, the ion fraction was calculated according to Eq. (3). The results obtained for polycrystalline Au were found to be $\sim 50 \%$ above the experimental data. Uncertainties in the external parameters (DOS, effective number of electrons, work function) do not have a sufficiently high impact on the resulting 
AN rate to explain this discrepancy. However, it turned out that $\Gamma$ reacts very sensitively to an energy shift of the He 1s level. In the calculations presented in Fig. 1, the He level was set to $-17.5 \mathrm{eV}$ w.r.t. to $E_{\mathrm{F}}$, which corresponds to a constant upward shift by $2 \mathrm{eV}$ (assuming a work function for $\mathrm{Au}$ of $5.1 \mathrm{eV}$, in good agreement with experimental values ${ }^{50}$ ). In comparison, calculations with the $\mathrm{He} 1 \mathrm{~s}$ level at $-21.0 \mathrm{eV}$ w.r.t. $E_{\mathrm{F}}$ result in an increase in $\Gamma$ for the on-top position by more than $40 \%$ at all projectile-surface distances. Calculations of $\mathrm{P}^{+}$for $\mathrm{He}^{+}$scattered from polycrystalline Au obtained by the "hard wall" and the "He-Al" level shift models (see Fig. 2) are presented in Fig. 3. Both subfigures contain experimental data (open asterisks) and corresponding single-exponential fits, $\exp \left(-v_{c} / v_{\perp}\right)$. In addition theoretical results calculated with a constant upward shift of the He level by $2 \mathrm{eV}$ are included (squares). Figure 3(a) presents the results obtained with the hard-wall level shift. Here, best agreement between theory and experiment is achieved when the He level is positioned at

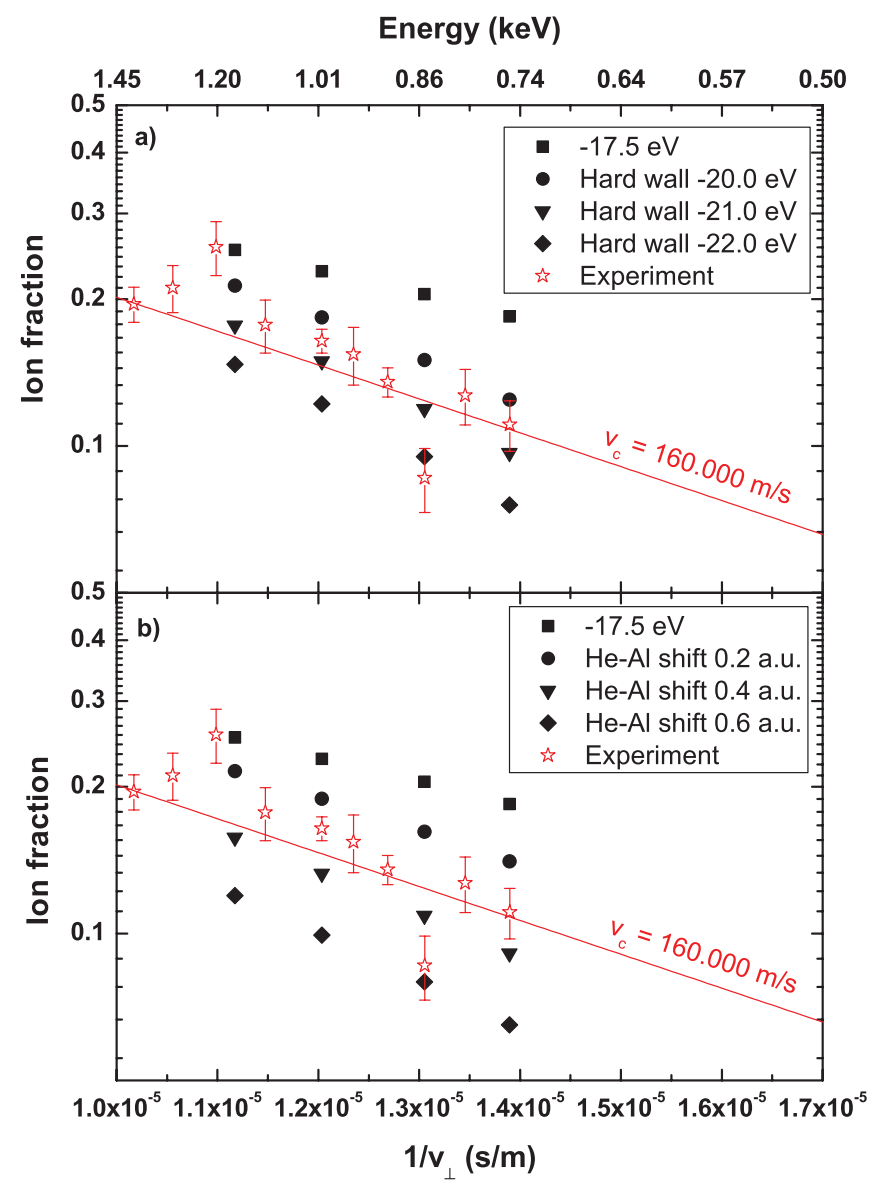

FIG. 3. (Color online) Ion fraction of $\mathrm{He}^{+}$scattered from polycrystalline $\mathrm{Au}$. Open asterisks correspond to experimental data. ${ }^{26}$ The straight lines correspond to a single-exponential fit. In both figures the results for the calculations, including a constant upward shift of the He level by $2 \mathrm{eV}(-17.5 \mathrm{eV})$, are shown (squares). Additionally, calculations are depicted (a) including the "hard wall" level shift with the He level set to $-20.0 \mathrm{eV}$ (circles), $-21.0 \mathrm{eV}$ (triangles), and $-22.0 \mathrm{eV}$ (diamonds) w.r.t. the Fermi level, and (b) including the "He-Al" level shift displaced towards the surface by a distance of 0.2 a.u. (circles), 0.4 a.u. (triangles), and 0.6 a.u. (diamonds), respectively.
$-21.0 \mathrm{eV}$ w.r.t. $E_{\mathrm{F}}$. The results obtained with the He-Al level shift model are depicted in Fig. 3(b). In this case very good agreement is obtained for a displacement of the $\mathrm{He}-\mathrm{Al}$ shift by 0.4 a.u. toward the surface (continuous line in Fig. 2). Thus, for both models best concordance is obtained for the same value for $r_{0}$, i.e., $r_{0}=0.6$ a.u. Note that this value for $r_{0}$ is very close to the experimentally deduced critical distance where AN breaks down and reionization sets in (0.61 a.u.). When comparing these two models, the hard-wall level shift can be interpreted as an average of a realistic level shift at distances where AN occurs. Consequently further calculations were performed with the hard-wall level shift, which is more easily implemented. Since the $\mathrm{Au}(110)$ surface is reconstructed and there is a significant contribution to the ion fraction from the second atomic layer, ${ }^{26}$ results for $\mathrm{Cu}$ and $\mathrm{Ag}$ are discussed next.

For $\mathrm{Cu}$ three different crystal orientations were investigated: (100), (110), and polycrystalline $\mathrm{Cu}$. The aim was to see whether the model correctly reproduces the experimentally observed physical matrix effect. As a first step, the ion fractions were determined based on calculations of $\Gamma$ with a constant upward level shift by $2 \mathrm{eV}$. Depending on the work functions of the particular surfaces [(110) and (100): $4.6 \mathrm{eV}$, poly: $\left.5.1 \mathrm{eV}^{50,51}\right]$, the $2 \mathrm{eV}$ shift corresponds to a He level at $18.0 \mathrm{eV}$ or $17.5 \mathrm{eV}$ below $E_{\mathrm{F}}$, respectively. Similarly as for $\mathrm{Au}$, only qualitative agreement between theory and calculations was achieved: The relative differences between the different crystal faces are reproduced correctly, but calculated AN rates would have to be increased by more than $30 \%$ in order to achieve quantitative agreement with experimental data.

Figure 4 presents the experimental ion fractions (open symbols) of $\mathrm{He}^{+}$scattered from $\mathrm{Cu}(100)$ (squares), $\mathrm{Cu}(110)$ (circles), and polycrystalline $\mathrm{Cu}$ (triangles), and singleexponential fits. Calculations were performed with a hard-wall

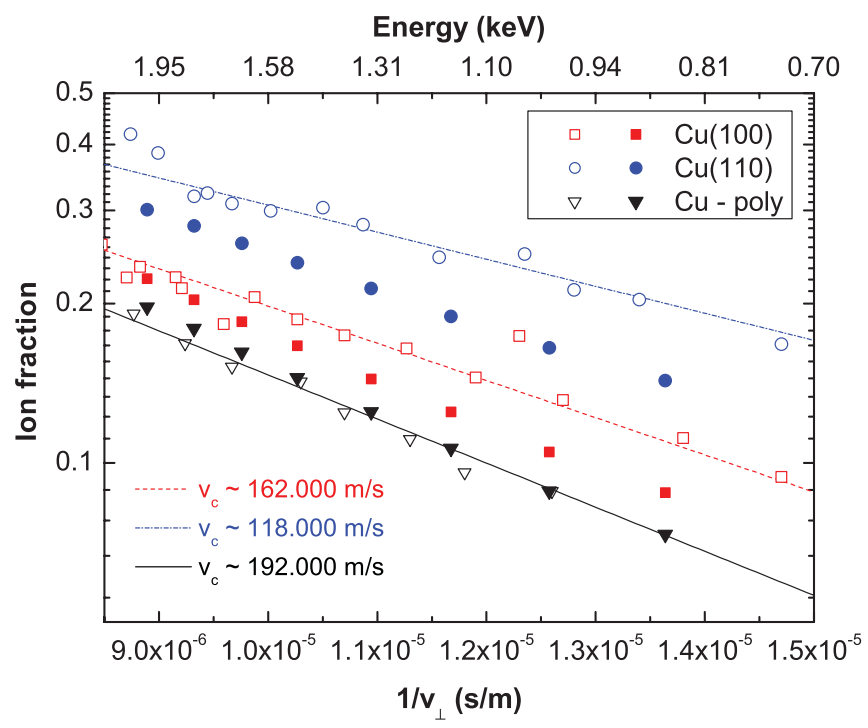

FIG. 4. (Color online) Ion fraction of $\mathrm{He}^{+}$scattered from $\mathrm{Cu}(100)$ (full squares), $\mathrm{Cu}(110)$ (full circles), and polycrystalline $\mathrm{Cu}$ (full triangles), calculated with a hard-wall level shift (He 1s level at $20.5 \mathrm{eV}$ below $E_{\mathrm{F}}$ ). Open symbols correspond to experimental data ${ }^{25}$; straight lines represent single-exponential fits. 


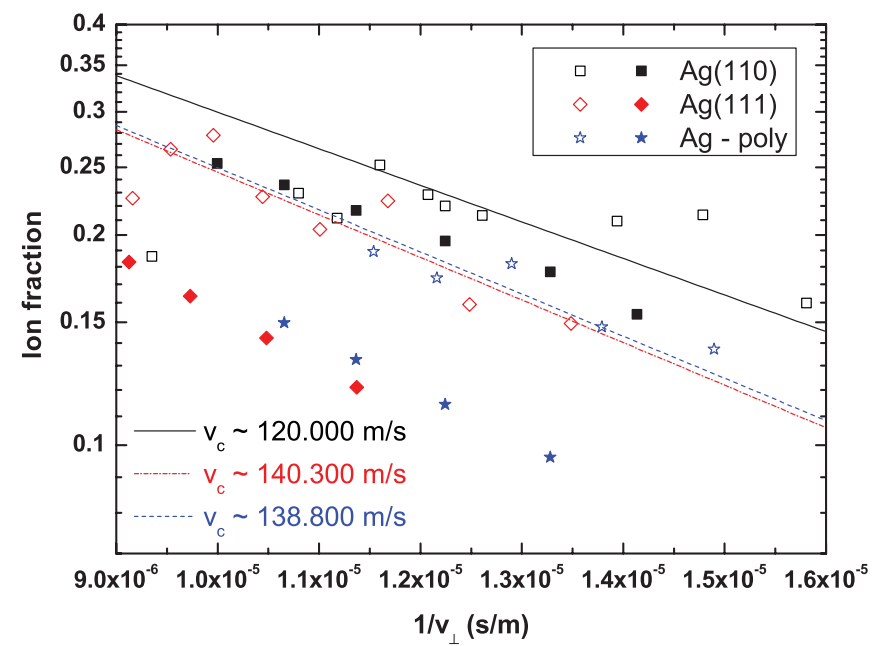

FIG. 5. (Color online) Ion fraction of $\mathrm{He}^{+}$scattered from $\mathrm{Ag}(110)$ (full squares), $\mathrm{Ag}(111)$ (full diamonds), and polycrystalline $\mathrm{Ag}$ (full asterisks), calculated with a constant upward shift of the He 1s level by $2 \mathrm{eV}$. Open symbols correspond to experimental data ${ }^{26,27}$; straight lines represent single-exponential fits.

level shift with the He level set to $-20.5 \mathrm{eV}$ w.r.t. $E_{\mathrm{F}}$ (full symbols). This calculation results in almost perfect agreement with the experimental data for polycrystalline $\mathrm{Cu}$. The same level shift also leads to very good agreement between theory and experiment for $\mathrm{Cu}(100)$ and fair agreement for $\mathrm{Cu}(110)$. For $\mathrm{Cu}(100)$ and $\mathrm{Cu}(110)$ the discrepancies may be due to uncertainties in the employed DOS and small differences in the level shifts for different surface orientations. In this respect it should be mentioned that for the He/Al system studied in grazing scattering geometry, ${ }^{24}$ the measured differences in level shifts for the different faces of $\mathrm{Al}$ are smaller than $1 \mathrm{eV}$, and one can expect even less differences in the present case of normal incidence. Note the remarkably similar level shifts used for $\mathrm{Cu}$ and $\mathrm{Au}$ surfaces, as might be expected in view of their similar band structures.

$\operatorname{Ag}(110)$ was already investigated previously, and good agreement between theory and experiment has been achieved. ${ }^{28}$ Figure 5 exhibits the results obtained for $\operatorname{Ag}(110)$ (squares), $\mathrm{Ag}(111)$ (diamonds), and polycrystalline Ag (asterisks). Full symbols correspond to calculated values, whereas open symbols represent experimental data. In the calculation the difference between $\mathrm{Ag}(111)$ and polycrystalline $\mathrm{Ag}$ arises from the different scattering geometry, which is used for $\operatorname{Ag}(111)$ in order to achieve maximum surface sensitivity. ${ }^{27}$ One can see that in contrast to the experimental findings, the calculated data exhibit a pronounced physical matrix effect. For $\mathrm{Ag}(111)$ and polycrystalline $\mathrm{Ag}$, the difference in $\mathrm{AN}$ rates amounts to $\sim 30 \%$, whereas for $\mathrm{Ag}(110)$ a discrepancy in $\Gamma$ of $\sim 10 \%$ can be deduced. These results were calculated with a constant upward shift of the He level by $2 \mathrm{eV}$. In contrast to the calculations for $\mathrm{Au}$ and $\mathrm{Cu}$, the calculated ion fraction for $\mathrm{Ag}$ is lower than the experimental data. Further investigations revealed that for silver $\Gamma$ reacts to an energy shift of the He level similarly, as $\mathrm{Au}$ and $\mathrm{Cu}$ do. Therefore, agreement between theory and experiment for $\mathrm{Ag}(111)$ would require to move $u p$ the energy level of He by about $4 \mathrm{eV}$.

From these findings for $\mathrm{Ag}$ it is possible to draw the following conclusions: Either the employed AN model does for some reason not describe Ag adequately, or the He level shift for Ag is fundamentally different from the level shifts of $\mathrm{He}$ for $\mathrm{Cu}$ and $\mathrm{Au}$. This latter assumption is supported by the investigation of $\mathrm{AN}$ for $\mathrm{Ag}$ in the grazing scattering regime. Here the calculated rates for $\operatorname{Ag}(111)$ have proven to be in accordance with experimental values. ${ }^{23}$ The difference in the level shift at close distances for $\mathrm{Au}$ and $\mathrm{Ag}$ could be due to the different positions of the $d$-bands. The surface state of $\operatorname{Ag}(111)$ should not lead to the observed differences, since Sarasola et al. found an increased AN rate due to presence of the surface state. ${ }^{52}$

\section{CONCLUSIONS AND OUTLOOK}

Based on the performed investigations, one can see that the influence of the electronic structure of a metal on AN of $\mathrm{He}^{+}$is twofold. Features of the DOS and of the atomic states influence the AN rate directly [see Eq. (1)] and indirectly via the He 1s level shift.

The results obtained for noble metals have shown that for these materials AN is governed by $d$-orbitals. Moreover the calculated rates indicate that in principle similar electronic structures lead to similar AN behaviors. The He 1s level turned out to have a significant influence on the resulting $A N$ rate. To a decrease in the energy of the He 1s level, all three noble metals reacted with an increase of AN rates in a similar way. This behavior of the noble metals is in contrast to that of $\mathrm{Al}$, for which calculations result in a decrease in $\Gamma$ when the $\mathrm{He}$ $1 \mathrm{~s}$ level is lowered by a specific amount. ${ }^{21}$ While calculations using a very similar He 1s level shift reproduce experimental results for $\mathrm{Cu}$ and $\mathrm{Au}$ quantitatively, investigations for $\mathrm{Ag}$ indicate a significantly different behavior. Good agreement between the employed model and results in grazing scattering support the hypothesis that for Ag the He 1s level shift at small ion-surface distances is significantly different than for $\mathrm{Au}$ or $\mathrm{Cu}$.

The impact of the He level shift on AN was obtained by use of two different models, each containing a free parameter, which could be fixed within physically reasonable limits. In order to gain further insight and give a definite answer to the puzzling behavior of Ag, detailed calculations of the level shift for the investigated noble metal surfaces are required.

\section{ACKNOWLEDGMENTS}

Support by the Austrian Science Fund FWF (projects P20831 and P19595) and the Spanish Ministerio de Ciencia e Innovación (project FIS2008-04209) is gratefully acknowledged.

\footnotetext{
*dominik.goebl@jku.at

${ }^{1}$ H. S. W. Massey, Proc. Cambridge Philos. Soc. 26, 386 (1930).

${ }^{2}$ A. Cobas and W. E. Lamb, Phys. Rev. 65, 327 (1944).
}

${ }^{3}$ H. D. Hagstrum, Phys. Rev. 96, 336 (1954).
${ }^{4}$ A. Zwartkruis and T. Fondén, Surf. Sci. 290, 134 (1993).
${ }^{5}$ R. Monreal and N. Lorente, Phys. Rev. B 52, 4760 (1995). 
${ }^{6}$ M. Alducin, Phys. Rev. A 53, 4222 (1996).

${ }^{7}$ N. Lorente and R. Monreal, Surf. Sci. 370, 324 (1997).

${ }^{8}$ M. A. Cazalilla, N. Lorente, R. D. Muino, J. P. Gauyacq, D. Teillet-Billy, and P. M. Echenique, Phys. Rev. B 58, 13991 (1998).

${ }^{9}$ J. C. Lancaster, F. J. Kontur, G. K. Walters, and F. B. Dunning. Phys. Rev. B 67, 115413 (2003).

${ }^{10}$ B. van Someren, P. A. Zeijlmans van Emmichoven, and A. Niehaus, Phys. Rev. A 61, 022902 (2000).

${ }^{11}$ M. Alducin, J. I. Juaristi, R. Díez Muiño, M. Rösler, and P. M. Echenique, Phys. Rev. A 72, 024901 (2005).

${ }^{12}$ R. Dirsyte, J. Schwarzkopf, G. Wagner, J. Lienemann, M. Busch, H. Winter, and R. Fornari, Appl. Surf. Sci. 255, 8685 (2009).

${ }^{13}$ H. Winter, Phys. Rep. 367, 387 (2002).

${ }^{14}$ H. H. Brongersma, M. Draxler, M. de Ridder, and P. Bauer, Surf. Sci. Rep. 62, 63 (2007).

${ }^{15}$ H. Niehus, W. Heiland, and E. Taglauer, Surf. Sci. Rep. 17, 213 (1993).

${ }^{16}$ R. Cortenraad, A. W. Denier van der Gon, H. H. Brongersma, G. Gärtner, and A. Manenschijn, Appl. Surf. Sci. 146, 69 (1999).

${ }^{17}$ L. C. A. van den Oetelaar, H. E. van Benthem, J. H. J. M. Helwegen, P. J. A. Stapel, and H. H. Brongersma, Surf. Interface Anal. 26, 537 (1998).

${ }^{18}$ N. P. Wang, E. A. Garcia, R. C. Monreal, F. Flores, E. C. Goldberg, H. H. Brongersma, and P. Bauer, Phys. Rev. A 64, 012901 (2001).

${ }^{19}$ R. C. Monreal, L. Guillemot, and V. A. Esaulov, J. Phys. Condens. Matter 15, 1165 (2003).

${ }^{20}$ Yu. Bandurin, V. A. Esaulov, L. Guillemot, and R. C. Monreal, Phys. Rev. Lett. 92, 017601 (2004).

${ }^{21}$ D. Valdés, E. C. Goldberg, J. M. Blanco, and R. C. Monreal, Phys. Rev. B 71, 245417 (2005).

${ }^{22}$ D. Valdés, J. M. Blanco, V. A. Esaulov, and R. C. Monreal, Phys. Rev. Lett. 97, 047601 (2006).

${ }^{23}$ D. Valdés, J. M. Blanco, V. A. Esaulov, and R. C. Monreal, Phys. Rev. B 75, 165404 (2007).

${ }^{24}$ S. Wethekam, D. Valdés, R. C. Monreal, and H. Winter, Phys. Rev. B 78, 075423 (2008).

${ }^{25}$ D. Primetzhofer, S. N. Markin, J. I. Juaristi, E. Taglauer, and P. Bauer, Phys. Rev. Lett. 100, 213201 (2008).

${ }^{26}$ D. Primetzhofer, M. Spitz, S. N. Markin, E. Taglauer, and P. Bauer, Phys. Rev. B 80, 125425 (2009).

${ }^{27}$ D. Primetzhofer, S. N. Markin, J. I. Juaristi, E. Taglauer, and P. Bauer, Nucl. Instr. Meth. B 267, 624 (2009).
${ }^{28}$ D. Goebl, R. C. Monreal, D. Valdés, D. Primetzhofer, and P. Bauer, Nucl. Instr. Meth. B 269, 1296 (2011).

${ }^{29}$ P. O. Löwdin, J. Chem. Phys 18, 365 (1950).

${ }^{30}$ B. R. Cooper, H. Ehrenreich, and P. Philipp, Phys. Rev. 138, A494 (1965).

${ }^{31}$ H. Ehrenreich and P. Philipp, Phys. Rev. 128, 1622 (1962).

${ }^{32}$ D. Beaglehole, Proc. Phys. Soc. 87, 461 (1966).

${ }^{33}$ P. Jelínek, H. Wang, J. P. Lewis, O. F. Sankey, and J. Ortega, Phys. Rev. B 71, 235101 (2005).

${ }^{34}$ J. P. Lewis, K. R. Glaesemann, G. A. Voth, J. Fritsch, A. A. Demkov, J. Ortega, and O. F. Sankey, Phys. Rev. B 64, 195103 (2001).

${ }^{35}$ O. F. Sankey and D. J. Niklewski, Phys. Rev. B 40, 3979 (1989).

${ }^{36}$ P. Nordlander and J. C. Tully, Phys. Rev. Lett. 61, 990 (1988).

${ }^{37}$ A. G. Borisov, D. Teillet-Billy, and J. P. Gauyacq, Nucl. Instr. Meth. B 78, 49 (1993).

${ }^{38}$ J. Merino, N. Lorente, P. Pou, and F. Flores, Phys. Rev. B 54, 10959 (1996).

${ }^{39}$ P. Kürpick, U. Thumm, and U. Wille, Phys. Rev. A 56, 543 (1997).

${ }^{40}$ S. A. Deutscher, X. Yang, and J. Burgdörfer, Phys. Rev. A 55, 466 (1997).

${ }^{41}$ H. Jouin, F. A. Gutierrez, and C. Harel, Surf. Sci. 417, 18 (1998).

${ }^{42}$ W. More, J. Merino, R. Monreal, P. Pou, and F. Flores, Phys. Rev. B 58, 7385 (1998).

${ }^{43}$ S. Wethekam, H. Winter, D. Valdés, and R. C. Monreal, Phys. Rev. B 79, 195408 (2009).

${ }^{44}$ S. Wethekam and H. Winter, Surf. Sci. 596, L319 (2005).

${ }^{45}$ Y. Bandurin, V. A. Esaulov, L. Guillemot, and R. C. Monreal, Phys. Rev. Lett. 92, 017601 (2004).

${ }^{46}$ M. A. Karolewski, Nucl. Instr. Meth. B 230, 402 (2005).

${ }^{47}$ J. Ziegler, J. Biersack, and U. Littmark, The Stopping and Range of Ions in Matter, Vol. 1 (Pergamon Press, New York, 1985).

${ }^{48}$ D. Chatain, V. Ghetta, and P. Wynblatt, Interface Science 12, 7 (2004).

${ }^{49}$ M. Draxler, S. N. Markin, S. N. Ermolov, K. Schmid, C. Hesch, R. Gruber, A. Poschacher, M. Bergsmann, and P. Bauer, Vacuum 73, 39 (2004).

${ }^{50}$ H. Kawano, Prog. Surf. Sci. 83, 1 (2008).

${ }^{51}$ P. O. Gartland, S. Berge, and B. J. Slagsvold, Phys. Rev. Lett. 28, 738 (1972).

${ }^{52}$ A. Sarasola, V. M. Silkin, and A. Arnau, Phys. Rev. B 75, 045104 (2007). 